\title{
Good drivers: Achieving dose reduction across a health care system through implementation of multiple radiation-sparing practices
}

\author{
Mathew Mercuri, PhD, ${ }^{\mathrm{a}}$ and Andrew J. Einstein, $\mathrm{MD}, \mathrm{PhD}^{\mathrm{b}}$ \\ a Department of Medicine, Division of Emergency Medicine, McMaster University, Hamilton, \\ ON \\ b Department of Medicine, Division of Cardiology, and Department of Radiology, Columbia \\ University Irving Medical Center and New York-Presbyterian Hospital, New York, NY
}

Received Jan 13, 2019; accepted Jan 14, 2019

doi: $10.1007 / \mathrm{s} 12350-019-01609-y$

\section{See related article, pp. 785-794}

Myocardial perfusion imaging (MPI) is a valuable diagnostic and prognostic tool for managing the care of cardiac patients. However, nuclear MPI modalities require the use of radiopharmaceuticals that expose patients to ionizing radiation, which is thought to be associated with a small but nonzero risk. In most cases, and in particular for appropriately indicated studies, the clinical utility of MPI far outweighs the potential harm to the patient of such radiation. Even so, optimizing the care of patients warrants ensuring that the resultant radiation exposure to the patient from ionizing radiation emitting modalities is kept to a level "as low as reasonably achievable,' to maximize the benefit-to-risk ratio. The concern for optimizing radiation dose (i.e., finding a balance between image quality, the utility of the imaging study, and radiation exposure to the patient) in diagnostic imaging, including MPI, has prompted substantial effort and interest from healthcare stakeholders. Over the past decade, that effort has led to the identification and encouragement of "best practices" and multiple awareness campaigns (e.g., Image Wisely

Reprint requests: Andrew J. Einstein, MD, PhD, Department of Medicine, Division of Cardiology, and Department of Radiology, Columbia University Irving Medical Center and New York-Presbyterian Hospital, PH 10-203, New York, NY; andrew.einstein@columbia.edu

J Nucl Cardiol 2020;27:795-7.

1071-3581/\$34.00

Copyright (C) 2019 American Society of Nuclear Cardiology. and Choosing Wisely) targeted at radiation dose reduction. In this issue of the Journal, Al Badarin et al. ${ }^{1}$ present the experience of a large, multicenter clinical group in their efforts over time to reduce ionizing radiation exposure to patients undergoing MPI in 9 sites of the Mid America Heart Institute health system in metropolitan Kansas City and its surrounding rural communities.

Recently, the International Atomic Energy Agency (IAEA) outlined eight best practices for optimizing radiation exposure in MPI procedures. ${ }^{2}$ These practices relate to radiopharmaceutical selection and dosing, protocol selection, and imaging technology (hardware and software) used. Empirical data support the notion that the use of these practices can result in a reduction in radiation exposure to patients undergoing MPI. For example, the recent INCAPS study highlighted the association between the use of each of these practices and reduced exposure to patients as measured by the effective dose. In the context of the United States, we also have empirical evidence that the use of these practices can result in relatively lower exposure to patients. ${ }^{3}$ For example, Chang et al. ${ }^{4}$ showed that the use of stress-only protocol was associated with a $61 \%$ reduction in radiopharmaceutical dosage, and thus a reduction in radiation exposure. Likewise, studies utilizing PET scanning were also shown to result in a lower dose $(3.7 \mathrm{mSv}$ vs. $12.3 \mathrm{mSv}$ mean effective dose for SPECT imaging MPI). ${ }^{5}$ Laboratories employing best practices are more successful at achieving a lower dose on average to their patient populations and are more likely to achieve the target of $\leq 9 \mathrm{mSv}$ effective dose, recognized as an important benchmark for radiation 
protection, when compared to laboratories where such best practices are not employed. ${ }^{2}$

The clinical practices examined by Al Baldarin et al. ${ }^{1}$ adopted several "radiation-sparing practices" (RSPs), similar to those outlined by the IAEA. The uptake of such RSPs differed among centers, although most of the over 55,000 patients observed had their studies performed at sites considered to have "advanced" capabilities for RSPs. Unsurprisingly, the investigators were able to show that the use of each RSP was associated with a reduction in estimated radiation dose to the patient. The greatest benefit was seen in studies that used a combination of stress-only protocol and modern imaging technology (e.g., solid-state SPECT camera, advanced post-processing software). Substantial reductions in the estimated radiation dose to the patient were also achieved with the use of PET and with the elimination of studies using thallium. Al Baldarin et al. ${ }^{1}$ also showed that laboratories with advanced capabilities for RSPs were more likely to achieve the target set by the American Society of Nuclear Cardiology (ASNC) of a median patient ED of $\leq 9 \mathrm{mSv}$. That is, laboratories utilizing the RSPs had a higher proportion of patients meet the benchmark for high performance in radiation protection when compared to laboratories that were less successful in implementing one or more of the RSPs $(65.7 \%$ vs. $10.8 \%)$.

The observed reductions in radiation exposure realized by the clinical practices examined by Al Baldarin et al. ${ }^{1}$ are encouraging. However, the results achieved by those clinicians are also something of an anomaly in the context of MPI practice in the United States. Data submitted to accreditation bodies around the same time as the cases observed by Al Baldarin and colleagues suggest a large majority of laboratories in the United States are exceeding the benchmark ED of $\leq 9 \mathrm{mSv}$ for MPI cases. ${ }^{6-9}$ Similar results were observed in the United States laboratories that participated in the INCAPS study. ${ }^{5}$ The INCAPS study also showed that the majority of those participating laboratories had yet to implement many of the radiation dose optimization best practices identified by the IAEA.

How is it that the those laboratories observed by $\mathrm{Al}$ Baldarin et al. ${ }^{1}$ were able to achieve what others in the United States could not with respect to radiation protection of patients undergoing MPI? The success realized by that group highlights the importance of resources devoted to dose reduction, in particular newer, more sensitive camera technology, and advanced postprocessing software that can result in diagnostic-quality images with lower administered activity. Certainly, one cannot expect that all laboratories will be able to implement these technological advances today, as the capital costs may be prohibitive. However, the cameras currently employed in many laboratories will age and eventually require replacement, at which point we could see radiation exposure to patients come down across the United States as a consequence of optimal use of newly acquired technology which is more dose-efficient.

The study by $\mathrm{Al}$ Baldarin et al. ${ }^{1}$ also highlights that improvements in radiation protection can be realized without the need for new and perhaps prohibitively expensive technology. For example, stress-only imaging can be used to reduce radiation dose to many patients with no additional resource requirements. ${ }^{10}$ Likewise, radiation dose reductions can be achieved with a change in the dose and in some case the type of the radiopharmaceutical(s) used in the MPI study protocol, with no appreciable loss of diagnostic utility. Such practices may even require fewer resources than are already devoted to MPI practice in many laboratories. However, implementing such strategies (so that the radiation dose reductions attributable to them may be obtained) may require a change in workflow or remuneration schemes. For example, stress-only MPI requires a physician to review images after the initial stress images are acquired so that a decision can be made about the need for rest imaging. In practices where a physician must attend to other tasks over the course of a clinic day, and as a result may only interpret the MPI studies at the end of the day, the use of a stress first protocol may require a change not only to activities related to MPI, but also to the cardiology practice at that clinic or hospital. Likewise, with some payers eliminating the rest portion of the MPI study may result in significant loss of income to the clinic, which in some cases may make the operation of the laboratory not financially viable (due to minimum personnel, infrastructure, and equipment costs required to operate the laboratory that must be amortized over fewer studies). One can surmise that low-volume laboratories and/or those located in smaller metropolitan or rural areas may be more impacted by this loss of income affecting the viability of the practice, potentially further exacerbating access inequities across the country. A possible solution may be to ensure that remuneration schemes do not penalize the laboratory financially for eschewing the often unnecessary rest imaging study (i.e., remuneration is tied to the activity of diagnosing the patient's pathology and not to the number of studies performed to reach that diagnosis). Changes in radiopharmaceutical use may also require changes in workflow, in particular to the order and timing of studies for a single patient.

Even if laboratories do not have the resources to acquire the latest camera technology or are bound to workflow and/or remuneration schemes, there are still 
ways to reduce in which radiation dose may be optimized. For example, Bloom and Meyers, ${ }^{11}$ interestingly from another large cardiology group in Kansas City, present some innovative strategies for radiation dose reduction to patients undergoing MPI where resources are limited. Those authors suggest the potential benefit of using a protocol with multiple positions during the stress phase of imaging and the use of advanced post-processing software that can be added onto existing software at a nominal cost. Their experience highlights the importance of innovation in resource-constrained environments. Certainly, those practices suggested by the IAEA for radiation protection are not exhaustive of all practices. It is incumbent on the nuclear cardiology community that it continues to seek out new ways of protecting patients while delivering high-quality care.

In most cases, potential detrimental effects of exposure to medical radiation are strongly outweighed by the clear benefits that can be realized from knowledge of pathology. The challenge to the clinician and patient alike is optimally balancing those benefits and risks. A reduction in radiation exposure, when it has no impact on the diagnostic utility of the study, may appreciably reduce risk with no impact on the realized benefit. Where that is the case, patients and physicians can perhaps feel more confident with a decision to use MPI in the management of care. Employing those best practices shown to reduce radiation exposure to the patient, as has been splendidly achieved in the Mid America Heart Institute health system, can help the community attain a population reduction in risk. Where those best practices cannot be employed, stakeholders should still seek innovative ways to reduce radiation exposure while maintaining diagnostic image quality. Of course, optimizing dose for any procedure is only one part of a comprehensive radiation protection strategy-any program to optimize radiation dose begins with conscientious use of imaging studies.

\section{Disclosure}

Dr. Einstein has served as a consultant to GE Healthcare and the International Atomic Energy Agency, and received grant funding to Columbia University from Canon Medical Systems, Roche Medical Systems, and the International Atomic Energy Agency. Dr. Mercuri has no relevant disclosures.

\section{References}

1. Al Baldarin FJ, Spertus JA, Bateman TM, Patel KK, Burgett EV, Kennedy KF, Thompson RC. Drivers of radiation dose with myocardial perfusion imaging: a large health care system experience. J Nucl Cardiol 2019; JNC-18-398-OA.R1.

2. Einstein AJ, Pascual TN, Mercuri M, Karthikeyan G, Vitola JV, Mahmarian JJ, et al. Current worldwide nuclear cardiology practices and radiation exposure: results from the 65 country IAEA Nuclear Cardiology Protocols Cross-Sectional Study (INCAPS). Eur Heart J. 2015;36:1689-96.

3. Thompson RC, O'Keefe JH, McGhie AI, Bybee KA, Thompson EC, Bateman TM. Reduction of SPECT MPI Radiation dose using contemporary protocols and technology. JACC Cardiovasc Imaging. 2018;11:282-3.

4. Chang SM, Nabi F, Xu J, Raza U, Mahmarian JJ. Normal stressonly versus standard stress/rest myocardial perfusion imaging: similar patient mortality with reduced radiation exposure. J Am Coll Cardiol. 2010;55(3):221-30.

5. Mercuri M, Pascual TNB, Mahmarian JJ, Shaw LJ, Rehani MM, Paez D, Einstein AJ. Comparison of radiation doses and bestpractice use for myocardial perfusion imaging in US and non-US Laboratories; Findings from the IAEA (International Atomic Energy Agency) Nuclear Cardiology Protocols Study. JAMA Intern Med. 2016;176:266-9.

6. Jerome SD, Tilkemeier PL, Farrell MB, Shaw LJ. Nationwide Laboratory adherence to myocardial perfusion imaging radiation dose reduction practices: a report from the Intersocietal Accreditation Commission Data Repository. JACC Cardiovasc Imaging. $2015 ; 8: 1170-6$

7. Desiderio MC, Lundbye JB, Baker WL, Farrell MB, Jerome SD, Heller GV. Current status of patient radiation exposure of cardiac PET and SPECT myocardial perfusion imaging: a report from the Intersocietal Accreditation Commission Database. Circ Cardiovasc Imaging. 2018;11:e07565. https://doi.org/10.1161/ CIRCIMAGING.118.007565.

8. Biecker MD, Butler PF, Bhargavan-Chatfield M, Harkness BA, Metter D, MacFarlane CR, et al. Adult gamma camera myocardial perfusion imaging: diagnostic reference levels and achievable administered activities derived from ACR accreditation data. J Am Coll Radiol. 2016;13:688-95.

9. Einstein AJ. High radiation doses from SPECT myocardial perfusion imaging in the United States: a lingering problem. Circ Cardiovasc Imaging. 2018;11:e008383. https://doi.org/10.1161/C IRCIMAGING.118.008383.

10. Mercuri M, Pascual TNB, Mahmarian JJ, Shaw LJ, Dondi M, Paez $\mathrm{D}$, Einstein AJ. Estimating the reduction in the radiation burden from nuclear cardiology through use of stress-only imaging in the United States and Worldwide. JAMA Intern Med. 2016;176:26973.

11. Bloom SA, Meyers K. Reducing radiation to patients and improving image quality in a real-world nuclear cardiology laboratory. J Nucl Cardiol. 2017;24:1871-7.

Publisher's Note Springer Nature remains neutral with regard to jurisdictional claims in published maps and institutional affiliations. 